\title{
Performance Analysis of Different Routing Protocols Using Directional Antennas
}

\author{
Pengxuan Mao* \\ Institute of Software Chinese Academy of Sciences \\ Beijing, China \\ e-mail:mpxbs@126.com
}

\begin{abstract}
Most of routing protocols are designed based on omni-antennas. The existing routing protocols with omniantennas show good performances on adhoc ground network. For some other applications, such as inter-satellite communications, directional antennas are a better choice because directional antennas have advantages in long range transmission, energy-saving and reduction of radio interference. However, directional antenna may lead to the performance degradation of existing routing protocols because it introduces new problems such as hidden terminal and deafness problem, and different antenna sweeping schemes also complicate the schedules in physical layer. In this paper, we will make comparisons of different routing protocols including AODV, OLSR, Bellmanford with directional antennas based on metrics of throughput, endto-end delay and jitter. We will deeply investigate the main factors affecting routing performances, and also perform extensive simulations under different scenarios using Qualnet 6.0 software. The simulation results show that AODV outperforms the OLSR, bellmanford in random scenarios. OLSR is a good candidate in satellite networks with stable topology.
\end{abstract}

Keywords-directional antenna; adhoc; OLSR; QoS, qualnet

\section{INTRODUCTION}

Nowadays, the wireless communication ground networks often utilize omni-antennas which may cause radio interference and energy-consumption waste by transmitting radio frequency in all directions. The current routing protocols in ad hoc network naturally work well with omni-directional antennas. However, for some other applications, such as long-range inter-satellite link communications, the routing performance will be degraded by using omni-antennas, because the node using omniantennas causes large energy waste. In order to complete the routing signal interaction, the node has to emit the signals in all directions. It will increase the packet collision opportunity.and complicate the link schedule. So many satellites usually use directional antennas for inter-satellite communications. With the fast development of directional antenna technology, the benefit of directional antennas attracts more and more attention. The size of a directional antenna becomes smaller and the cost of it reduces as well.

The characteristic of radiating radio signal towards a direction makes satellite energy consumption reduction, enlarge communication range and reduce co-channel interference compared with omni-directional antennas. However, the utilization of directional antenna faces the challenges as well. In space satellite network, directional antennas introduce new hidden terminal and deafness problems when working with traditional MAC protocols , and also bring in new neighbor discovery problem and routing overhead problem when working with traditional routing protocols [6]. The problem we really concern is the effects on conventional routing protocols by utilizing directional antennas. Whether the traditional routing protocols' performance using directional antenna in satellite networks can satisfy the demanding of satellite data transmission without modifying the routing protocols themselves. Then we will fully investigate the potential factors affecting routing protocols including MAC mechanisms, link state, directional antennas' sweeping scheme and network topology.

This paper is organized as follows: Section II presents the related work done so far. Section III presents the description of routing protocols. Section IV represents the classification of directional antenna. Section V represents simulation scenarios. Section VI shows the results and make conclusions.

\section{RELATED WORK}

Recently, the benefits of directional antennas attract a lot of researchers' attentions. The authors[1] make the performance comparison between omni-antenna and directional antenna using different routing protocols. Their results show that the networks with directional antennas are able to achieve higher throughput. The researchers[2] proposed a modified CSMAICD medium control mechanism that utilizes a kind of directional antenna called switched beam antenna array. The authors[3] evaluated the traditional routing protocols' performance including AODV, LANMAR, RIP using three types of directional antennas compared with omni-antenna. The energy consumption of different routing protocols with directional antennas is investigated by Dharam Vir[4]. The results show that the OLSR consumes least energy in all directional antennas. The authors[5] make comparisons with three routing protocols (AODV,OLSR, GRP) using directional antennas in grid network scenarios. The results show that OLSR routing protocol have better performance and higher throughput as compared to AODV,GRP routing protocols. However, they didn't consider the fact of directional antennas' sweeping scheme. Some others take another approach as follows. Romit Roy Choudhury [6] points that the challenges the utility of directional antennas will face are from the view of MAC layer. The deafness problem need be considered. The results attract other researchers' attention. Yuya Takatsuka[7] analyzes 
the existing MAC mechanisms based on practical smart antennas array, and then propose a directional MAC mechanism which can achieve higher throughput. HiKaru MITSUHASHI[8] built a testbed with a practical smart antenna for directional MAC protocols in ad hoc networks. Han song[9] also focus on designing a particular MAC mechanism for directional antenna scenarios called $\mathrm{M}$ DMAC in order to improve the QoS of ad hoc network. However, these approaches above have to modify the basic MAC mechanisms, which complicate their implements in real networks. So, it's more feasible to find the optimal configuration parameters of routing protocols. In this paper, we will deeply investigate the main factors which affect the routing protocols' performance. We will perform extensive simulations with Qualnet 6.0 to evaluate the routing protocols in different scenarios.

\section{ROUTING PROTOCOLS}

In this section, we will discuss three kinds of conventional routing protocols which are designed for adhoc networks. The protocols are as following: AODV, OLSR, BELLFORD. In fact, there are mainly two approaches of building routes in ad hoc networks: the reactive routing approach and proactive routing approach. Both of them have advantages and drawbacks, and are well applicable in their respective places.

The reactive routing protocol discovers routes on demand by flooding information packets in the ad hoc network. The AODV (Ad hoc On Demand Distance Vector routing protocol) routing protocol is one of many reactive routing protocols which is defined in RFC 3561. AODV finds a new route by reducing the control traffic packet overheads and maintains route path information in nodes. The AODV is capable to provide self-adaptation to dynamic network topology, link error, and supports IPv4 stack. The AODV has some basic components such as routing table and message format. Similar to other distance vector routing algorithms, the AODV maintains Routing table to deliver packets. The AODV routing protocol maintains the optimal routing path towards any destination in the routing table for a configured period. When there are two nodes which want to transfer information, and no route path in Routing table, AODV will initiate the routing discovery procedure. AODV can also keep routing information fresh with a destination sequence number and avoid causing a routing loop by abandoning out of date route. AODV also has a high convergence speed when network topology is changing. The routing table stores one path for one destination. This path could forward packets fast and reliably, although the path may not be the shortest path. A source node can use AODV messages to maintain the newest active path for the source. The AODV messages mainly have RREQ, RREP, and RREP-ACK. RREQ is used ad broadcast packet when the source node initiates a path discovery procedure. An intermediate node which finds a fresh route to the destination sends a RREP message back to the requesting node. When a source node initiates communication and does not have a recent route to the destination then it starts two procedures, route discovery and route maintenance. Route discovery: The AODV routing protocol broadcasts the route request packet(RREQ) . If the neighboring or intermediate node which receives the RREQ has no route about the target destination, it will continue to broadcast RREQ packet in the network. Once the destination is found, it will send an answer key by the route reply (RREP) packet to the sender from which RREQ is received. When the RREP is received at the source node, the path is established. RREQ contains source address, source sequence number, broadcast id, destination address, destination sequence number and hop count [5]. Route maintenance: It is the next step which is followed by the AODV protocol after route discovery. In this step, it finds the error which comes during transmission like if the two nodes that were listed as neighbor on the route moved out of the range of each other and link is broken [1].

The proactive routing approach shares recent network topology information with all the nodes by periodically exchanging control messages. OLSR defined in RFC3626 is a proactive routing protocol. It maintains and updates network topology information for each node by exchanging periodical messages. Different to other classical link state approach which floods topology information to the entire network, the OLSR routing protocol takes more efficient approach to flood the control message by Multipoint Relay mechanism (MPR). First, OLSR performs neighbor discovery procedure by periodically exchanging HELLO messages between onehop neighbors with MPR algorithm. It computes the optimal route to each destination in the network for every node based on sufficient topology information. In OLSR, only an MRP node can be selected as relay node for routing packets. The route calculation is based on the information contained in the topology table and the neighbor table of each node. In OLSR, only an MRP node can be selected as relay node for routing packets. The route calculation is mainly based on the information contained in the topology table and the neighbor table of each node.

The ZRP routing protocol is based on hybrid routing approach which take the advantages of both proactive and reactive routing approaches. The ZRP routing protocol spares bandwidths to maintain path information. The ZRP routing protocols are divided into two zones (outside and inside). Due to a number of packets will be delivered to the near destination, the proactive routing protocol is used within the zone to reduce the topology maintenance costs in a limited zone. In addition, when the source and destination node are within the same zone, it will not introduce initial delay. While the reactive routing protocol is utilized outside the zone when sometimes there is packet to be routed to the remote destination outside the zone[11].

\section{DIRECTIONAL ANTENNAS}

In this section, we will discuss the details of directional antennas. The omni-directional antenna radiates the electromagnetic energy in all directions. if nodes in a network are located within each others' transmission range, the communication link is established. 
However, the omni-directional antenna faces co-channel interference when simultaneous data transmission happens. The opportunities of packet collisions will be increased when the number of nodes becomes larger. The performance of routing will be degraded. Directional antenna can be used to avoid co-channel interference. There are two main types of directional antenna: mechanically switched and steerable antenna. Both of them has respective sweeping scheme. The details of the two directional antennas are as following:

1) Steerable Antenna is a typical directional antenna that has the capability of adapting the beam in a particular direction. The major lobe of Steerable antenna can be shifted readily in direction. a steerable antenna has capability to fix the the beam to a specific angle of the receiver node. Steerable antenna system have a logic combines the antenna elements in such a way that the beam is directed towards any given angle. The antenna elements are placed in such a way that main lobe, side lobe and tail lobe do not create interference and hence interference is reduced. The steerable antenna is a special type of patterned antenna. A patterned antenna has different gains in different directions. The values of the gain in different directions follow a gain pattern. The steerable antenna can rotate the antenna and uses the direction that yields the maximum antenna gain [4].

2) Switched beam: the switched beam antenna is the simplest smart antenna. It utilizes a number of fixed beams in preconfigured directions. The antenna selects the beam that supports the maximum SINR. It uses a radio frequency that combines the antenna elements. These antenna elements divide the network in equal sectors and antenna elements emit the fixed beam that covers one sector. In network when the nodes are moved then switched beam antenna propagates the fixed beam and provide the better performance. Switch beam antenna base station selects the beam that supports the maximum signal to interference and noise ratio. The main disadvantage of switch beam antenna is its fixed nature due to which it cannot focus at a specific angle [8].

\section{PERFORMANCE ANALYSIS}

In this section, we will perform simulations in different scenarios in Qualnet 6.0[10], and evaluate the QoS of different routing protocols with directional antenna.

we use the scenario size $900 * 900 \mathrm{~m}^{2}$, CBR of packet size is 512, the simulation time is $30 \mathrm{sec}$, physical protocol $802.11 \mathrm{~b}$ and PHY ABSTRACT. It analyzes the performance of AODV, OLSR and ZRP protocols using different Directional antennas. We will use three different scenarios including two types of gird topology and one topology with mobility. Fig. 1 shows grid topology (one subnet). In this scenario, each node has one interface to be installed one directional antenna. The topology is stable. Fig.2 shows the snapshot of Qualnet 6.0 simulation snapshot.

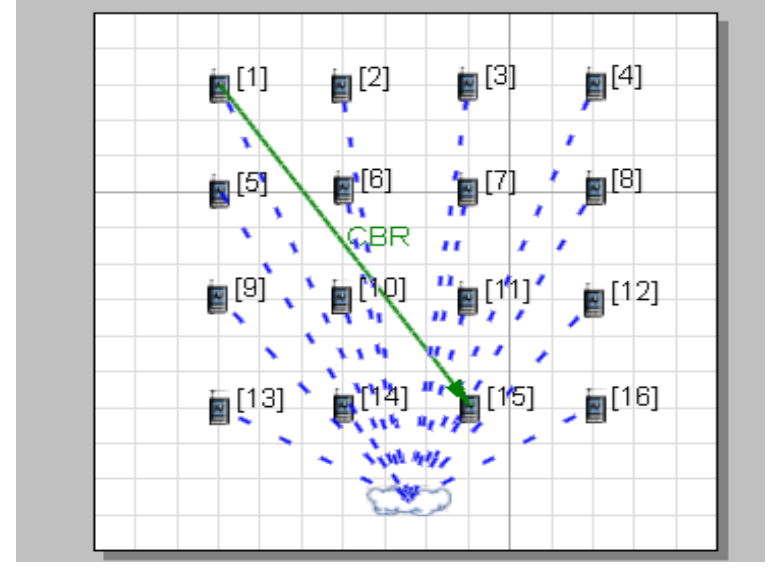

Figure 1. Grid network (one interface)

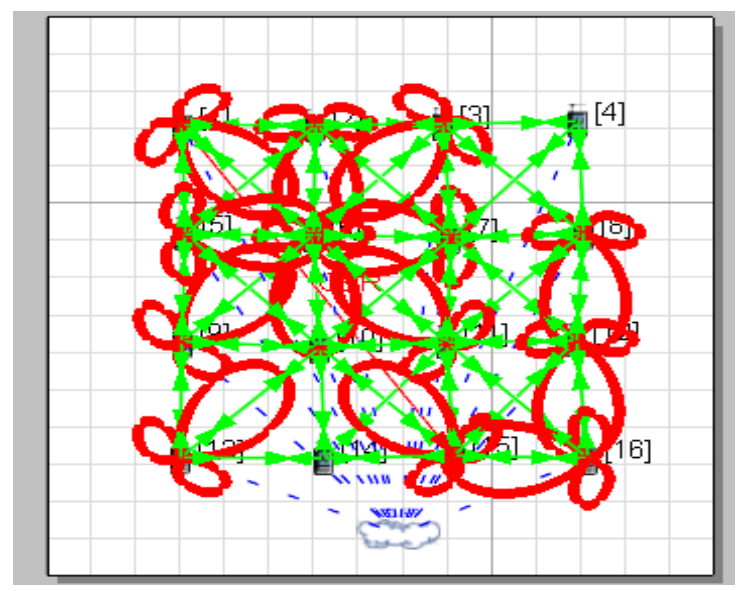

Figure 2. Simulation snapshot

The basic parameter configurations are as shown in Table. 1 .

TABLE I. PRAMETER CONFIGURATOIN

\begin{tabular}{|c|c|}
\hline Parameter & Value \\
\hline Simulation platform & Qualnet 6.0 \\
\hline Routing Protocols & AODV, OLSR,ZRP \\
\hline Physical protocols & $802.11 \mathrm{~b}$, abstract \\
\hline Simulation time & 30 seconds \\
\hline Antenna model & $\begin{array}{l}\text { Steerable Antenna } \\
\text { Switched Antenna }\end{array}$ \\
\hline Application Type & CBR, FTP \\
\hline Frequency & $2.4 \mathrm{GHz}$ \\
\hline Number of Nodes & 16 \\
\hline Link Type & Wireless Link \\
\hline QoS metrics & $\begin{array}{l}\text { Average Delay Jitter, } \\
\text { Average end-to-end } \\
\text { Delay, Throughput }\end{array}$ \\
\hline Source, Destination & 1,15 \\
\hline Item size & 512 bytes \\
\hline
\end{tabular}

We will investigate the QoS metrics of different routing protocols in different scenarios. The metrics are average end-to-end throughput, average end-to-end delay and delay. 


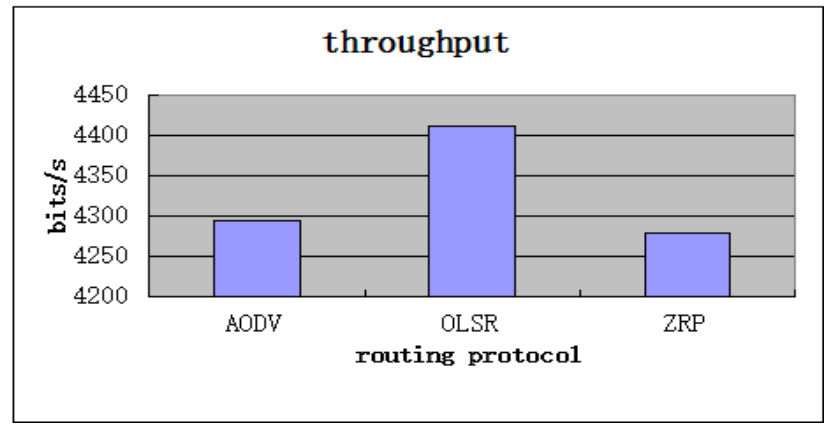

Figure 3. Throughput (one interface)

Fig. 3 shows the throughput comparison of AODV, OLSR and ZRP. The simulation results are as follows. The OLSR routing protocol with steerable antennas presents the largest throughput compared with AODV, ZRP as shown in Fig.3. The ZRP presents least value of the average jitter for all three routing protocols as shown in Fig.4. The AODV presents least value of the average end-to-end delay for all three routing protocols as shown in Fig.5. In this scenario, the performance of different routing protocols using steerable antennas and switch beam antenna is the same. We also use PHY_ABSTRACT. The results show that the throughput using PHY_ABSTRACT has almost the same as the one using $802.11 \mathrm{~b}$.

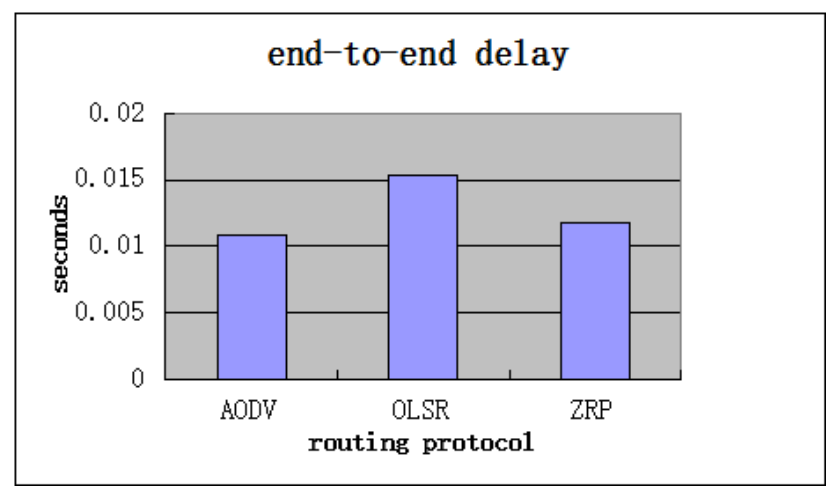

Figure 4. The end-to-end delay (one interface)

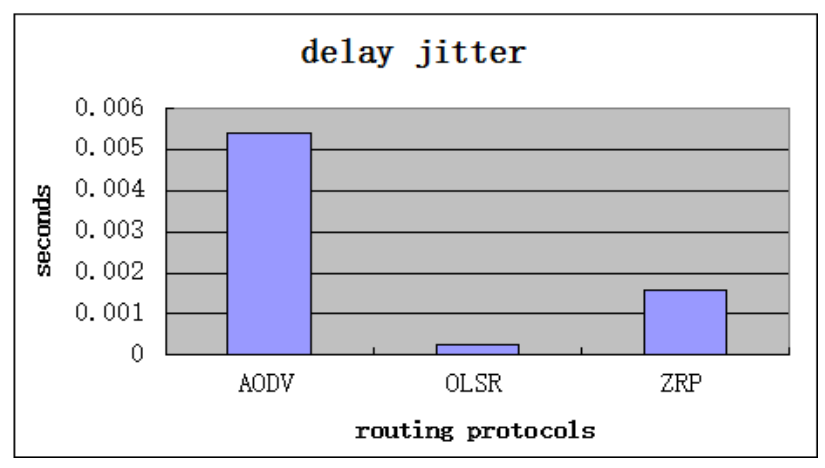

Figure 5. Delay jitter (one interface)
We will use the grid scenario as shown in Fig.6. Each node is installed four directional antennas. The topology is stable.

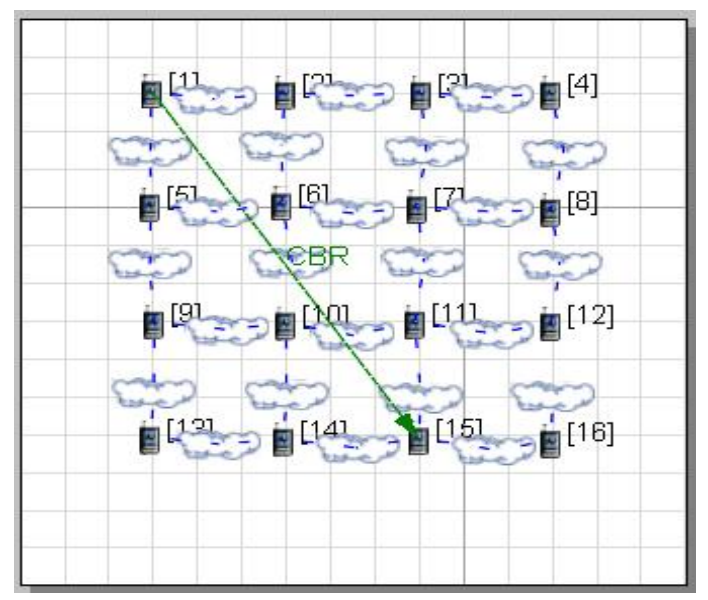

Figure 6. Grid network (four interface)

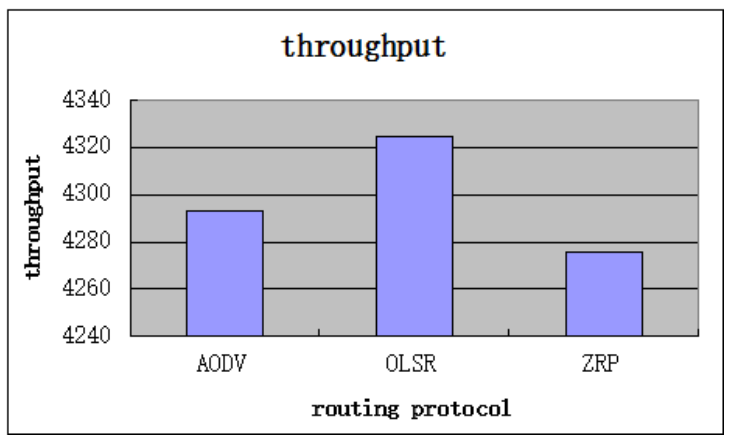

Figure 7. The throughput (four interfaces)

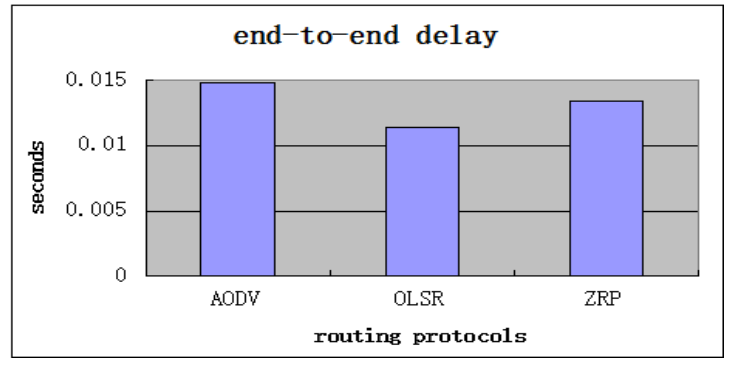

Figure 8. The end-to-end delay (four interfaces)

Fig.7 shows the throughput comparison of AODV, OLSR and ZRP in grid scenario as shown in Fig.6. The simulation results are as follows. The OLSR routing protocol with steerable antennas presents the largest throughput compared with AODV, ZRP as shown in Fig.7. The OLSR presents least value of the average end-to-end delay for all three routing protocols as shown in Fig.8.

We will use the random mobility scenario as shown in Fig.9. Each node is installed one steerable antenna. The source node can move in trace of red flags as shown in Fig.9. 


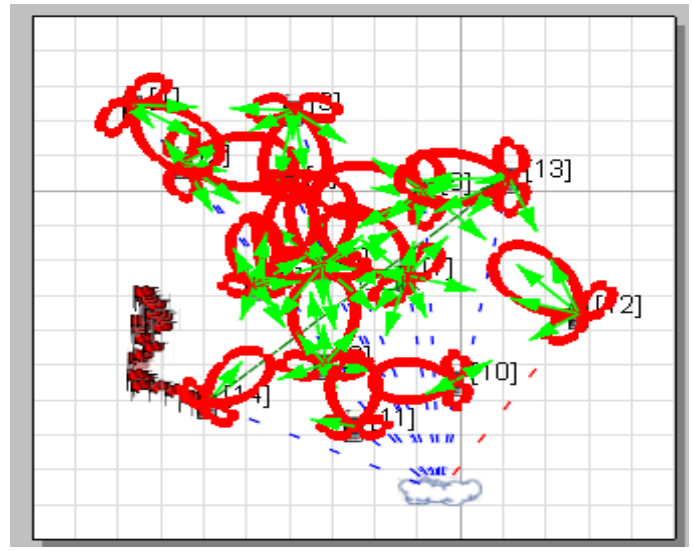

Figure 9. The random topology

Fig.10 shows the throughput comparison of AODV, OLSR in random topology as shown in Fig.9. The simulation results are as follows. The AODV routing protocol with steerable antennas presents the largest throughput compared with OLSR as shown in Fig.10.

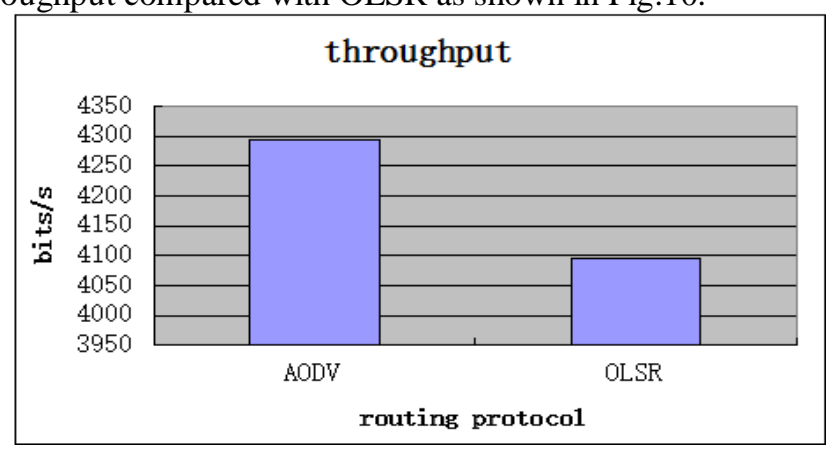

Figure 10. The throughput

\section{CONCLUSION}

In this paper, we perform comparison between different protocols (AODV, OLSR and ZRP) using different types of Directional antennas (Steerable and Switched Beam). We fully investigate the QoS metrics of different routing protocols. Finally we conclude that in relative stable topology, OLSR provides highest values for throughput as compared to AODV, ZRP. In random topology with mobility, AODV provides higher values for throughput as compared to OLSR.

\section{REFERENCES}

[1] Hao Zhou, "The Improvements in Ad Hoc Routing and Network Performance with Directional Antennas" Master Thesis, Helsinki University of Technology, 2006.

[2] Sultan Budhwani, "A MAC Layer Protocol for Sensor Networks using Directional Antennas", IEEE International Conference on Sensor Networks, Ubiquitous, and Trustworthy Computing,2011.

[3] Ankit Jinda, Charanjeet Singh "Simulation Analysis of Different Routing Protocols Using Directional Antenna in Qualnet 6.1 ", Vol. 3, Issue 5, May 2014

[4] Dharam Vir, "Performance Analysis Of Effect Of Directional Antennas On Energy In Routing Protocol”, International Journal of Engineering Research and Applications (IJERA), Vol. 3, Issue 1, January -February 2013, pp.238-244.

[5] Mandeep Singh, "Performance of AODV, GRP and OLSR Routing Protocols in Adhoc Network with Directional Antennas ", Volume 83 - No2, December 2013.

[6] Yuya Takatsuka, "A Directional MAC Protocol for Practical Smart Antennas ”, IEEE GLOBECOM 2006.

[7] Hikaru MITSUHASHI, "A Testbed with a Practical Smart Antenna for Directional MAC Protocols in Ad hoc Networks ",21st International Conference on Advanced Information Networking and Applications Workshops (AINAW'07) .

[8] Romit Roy Choudhury, "Deafness: A MAC Problem in Ad Hoc Networks when using Directional Antennas", INFOCOM, 2003.

[9] Han Song, “A Modified Directional MAC Protocol for using Smart Antenna in Wireless Ad hoc and Sensor Networks",

[10] “QualNet simulator model library”.

[11] B. Alawieh, C. Assi, W. Ajib, "A Power Control Scheme for Directional MIAC Protocols in MANET" , IEEE Conf, WCNC2007, pp.258-263, 2007. 